Trauma Berufskrankh 2004 - 6 [Suppl 2] : S273-S278 DOI 10.1007/s10039-003-0793-1

Online publiziert: 15. November 2003

(c) Springer-Verlag 2003

T. R. Blattert · A. Weckbach

Unfall- \& Wiederherstellungschirurgie, Chirurgische Universitätsklinik,Würzburg

\title{
Kalziumphosphat vs. Polymethylmethacrylat
}

\section{Erste Ergebnisse einer prospektiven, randomisierten, klinischen Vergleichsstudie zur perkutanen Ballonkyphoplastie}

\section{Anforderungsprofil für Zemente - Theoretische Vorbemerkungen}

\section{Viskosität und Röntgendichte}

Die Beimischung von 30-40 vol\% Röntgenkontrastmittel (Bariumsulfat usw.) zum in seiner nativen Form nicht ausreichend röntgendichten PMMA geht mit einer Reduktion der Viskosität einher, weswegen ein theoretisch höheres Risiko der Zementextrusion und/oder Gefäßembolisation bei Verwendung von PMMA besteht. Kalziumphosphatzement besitzt dagegen eine immanente Röntgendichtigkeit und bedarf somit keiner Beimischung verflüssigender Zusatzstoffe.

\section{Bearbeitungs-/Aushärtungszeit}

Bearbeitungszeit und Aushärtungszeit sind sowohl bei PMMA als auch bei Kalziumphosphat innerhalb eines weiten Bereichs einstellbar und somit für beide Stoffgruppen der Indikation entsprechend ideal wählbar. Hierbei erfolgt die Spezifikation der jeweiligen Bearbeitungs- und Aushärtungszeiten für PMMA meist durch Addition von Benzoylperoxid oder Hydroquinon, für Kalziumphosphat dagegen durch Beimischung von Glyzerin oder Polyethylenglykol. Zudem lässt sich für beide Gruppen durch Vorwahl der Anmischtemperatur eine breite Adaptation an die individuellen Bedürfnisse erreichen. Meist wird eine Bearbeitungszeit von etwa 10 min bei $23^{\circ} \mathrm{C}$ als optimal empfunden, wobei die Aushärtungszeit bei $37^{\circ} \mathrm{C}$ dann mit etwa $20-30$ min ebenfalls in einem geeigneten Bereich liegt.

\section{Aushärtungsverhalten}

Polymethylmethacrylat unterscheidet sich von Kalziumphosphat durch einen chemisch völlig andersartigen Aushärtungsprozess: Während sich PMMA im Rahmen einer exothermen Polymerisationsreaktion verfestigt, kommt es bei Zementen auf Kalziumphosphatbasis zu einer Kristallisation, die isotherm verläuft. Somit werden in der Aushärtungsphase von PMMA-Zementen Temperaturen von $60^{\circ} \mathrm{C}$ und mehr erreicht [9], während sich Kalziumphosphate bei Körpertemperatur verfestigen. Durch die hohe Temperaturentwicklung besteht daher für PMMA ein erhöhtes Risiko der thermischen Schädigung anatomisch benachbarter Strukturen, was bei Zementextrusionen - insbesondere nach epidural - weitreichende Folgen haben kann.

Ein ganz anderer Aspekt ergibt sich hingegen aus der Tatsache, dass Kalzium- mechanische Eignung und biologisches Verhalten. 

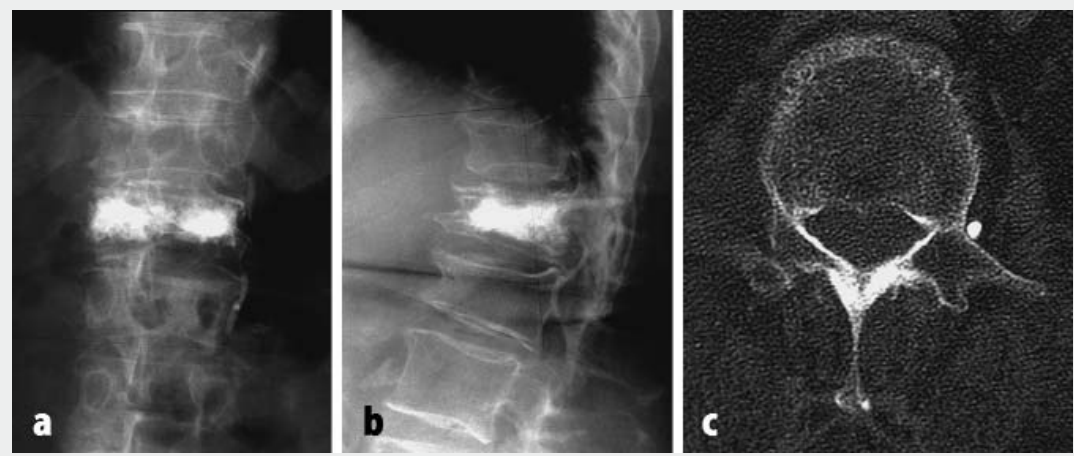

Abb. 1a-c $\Delta$ Thorakolumbaler Übergang 3 Tage nach perkutaner, bipedikulärer Ballonkyphoplastie L1 mit PMMA. Röntgen nativ a.-p. im Stehen (a), Röntgen seitlich im Stehen (b), axiale CT-Schicht auf Bandscheibenhöhe L1/L2 (c): deutlich erkennbare Zementembolisation der zugehörigen linken Segmentvene L1
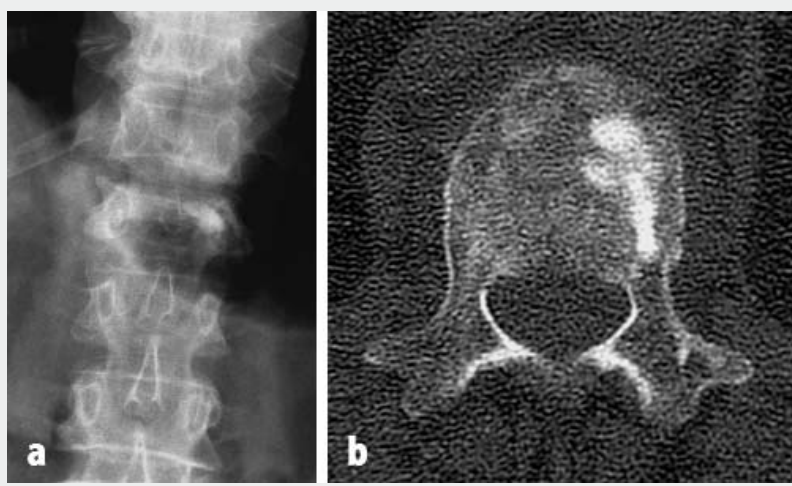

Abb. 2a,b $\Delta$ Thorakolumbaler Übergang 3 Tage nach perkutaner, bipedikulärer Ballonkyphoplastie L1 mit Kalziumphosphat, Röntgen nativ a.-p. im Stehen (a) und axiale CT-Schicht durch L1 (b): rechts vollständiges, links teilweises „Auswaschen“ des Zements durch intraoperativ anhaltende Blutung aus der Wirbelkörperspongiosa (injiziert wurden rechts $3 \mathrm{ml}$ und links $4 \mathrm{ml}$ Kalziumphosphatzement)

phosphat grundsätzlich ein geringeres spezifisches Gewicht aufweist als Zemente auf PMMA-Basis. Damit besitzt Kalziumphosphat eine im Vergleich zu PMMA theoretisch verminderte Widerstandskraft gegenüber einem ungewollten „Auswaschen" des Zements durch Blut während der Aushärtungsphase.

\section{Mechanische Eignung}

Ganz offensichtlich sind Zemente, die der Augmentation von Wirbelkörpern dienen, einer Kompression in axialer Belastungsrichtung ausgesetzt und müssen damit eine bestimmte Druckfestigkeit erreichen, um überhaupt geeignet zu sein. Es ist jedoch davon auszugehen, dass eine intrakorporell liegende Zementplombe durchaus in bestimmten Situationen auch wiederholte Biege-, Zug- und Scherkräfte erfährt, nämlich immer dann, wenn im Fall der Zerstörung des kortikalen Wirbelkörperrahmens diese Kräfte ungerichtet nach zentral weitergeleitet werden können.

Die physiologische Druckfestigkeit menschlicher Wirbelkörperspongiosa liegt im Bereich von 2-20 MPa [19]. Sowohl PMMA-Zemente mit einer mittleren Druckfestigkeit von $95 \pm 5 \mathrm{MPa}$ als auch Kalziumphosphate, die im Mittel bei $55 \pm$ 5 MPa Druckfestigkeit liegen, erreichen damit ohne weiteres die geforderte Widerstandskraft gegen Kompression in axialer Belastungsrichtung. Hingegen wird der empfohlene Standard für Zemente hinsichtlich ihrer Zug- und Biegefestigkeit von $\geq 30 \mathrm{MPa}[3]$ nur von PMMA erreicht $(37 \pm 4 \mathrm{MPa})$, nicht jedoch von Kal- ziumphosphaten $(3 \pm 0,7 \mathrm{MPa})$. Damit besteht theoretisch für Zemente auf Kalziumphosphatbasis ein mechanisches Versagensrisiko in all den Fällen, in denen relevante Biege-, Zug- und/oder Scherbeanspruchungen auf das Biomaterial einwirken können.

\section{Biologisches Verhalten}

Die osteokonduktiven Eigenschaften von Kalziumphosphat lassen eine graduelle Biointegration mit anschließender ossärer Substitution des Biomaterials und schließlich ein Remodelling der Wirbelkörperspongiosa erwarten. Dies stellt einen entscheidenden Vorteil gegenüber PMMA dar, welches langfristig bestenfalls als inerte Masse im Wirbelkörper verbleibt.

Ein weiterer Nachteil im biologischen Verhalten von Polymethylmethacrylat ist der u. U. unvollständig verlaufende Polymerisationsprozess, sodass toxische Monomere verbleiben und $u$. U. unerwünschte systemische Nebenwirkungen entfalten können $[1,6,21,23]$. Dem steht die aufgrund ihrer strukturellen Nähe zur originären Knochensubstanz uneingeschränkte Biokompatibilität von Kalziumphosphatprodukten gegenüber.

\section{Methode}

Im Rahmen einer prospektiven, randomisierten, klinischen Studie soll die Eignung von Kalziumphosphat zur Wirbelkörperaugmentation im Rahmen der perkutanen Ballonkyphoplastie überprüft und mit dem derzeitigen Zementstandard Polymethylmethacrylat verglichen werden. Als Einschlusskriterium wurde das Vorliegen osteoporotischer Wirbelkörperfrakturen im Bereich der thorakalen oder lumbalen Wirbelsäule definiert, und zwar unabhängig von Patientengeschlecht und -alter sowie von Frakturtyp und -alter. Ausschlusskriterien sind eine zusätzlich notwendige dorsale Instrumentierung sowie pathologische Frakturen durch Tumor- oder Metastasenbefall.

Die perkutane bipedikuläre Ballonkyphoplastie (Kyphon Inc., Sunnyvale, USA) wird in der Standardtechnik unter Intubationsnarkose in Bauchlage mit Verwendung zweier Bildwandler simultan jeweils in der a.-p.- und seitlichen Ebene durchge- 
führt. Der Randomversuchsarm beinhaltet die Augmentation des betroffenen Wirbelkörpers mit einem Kalziumphosphat-Karbonat-Zement (Norian SRS ${ }^{\mathrm{TM}}$, Norian Corp., Cupertino, USA), während im Randomkontrollarm ein Polymethylmethacrylatzement (Vertebroplastic ${ }^{\mathrm{TM}}$, DePuy Acromed, Raynham, USA) zum Einsatz kommt.

Die Studie ist als balanciert randomisierte Untersuchung konzipiert, wobei insgesamt 6o Wirbelkörperfrakturen eingeschlossen werden sollen. Nachuntersuchungen zur klinischen (körperliche Untersuchung, subjektive Schmerzbenotung von 1-6) und radiologischen (Nativröntgen in 2 Ebenen, CT axial in 2-mm-Schichten mit sagittaler Rekonstruktion) Befunderhebung sind für die folgenden Zeiträume vorgesehen: 3 Tage, 6 Wochen sowie 3, 9 und 24 Monate postoperativ.

\section{Ergebnisse}

Zum Zeitpunkt der vorliegenden Zwischenauswertung waren für den Zeitraum von August 2002-Mai 2003 insgesamt 19 Patienten mit 22 Wirbelkörperfrakturen eingeschlossen, darunter 18 Frauen und 1 Mann. Bei 3 Patienten waren jeweils 2 Wirbelkörper gleichzeitig behandelt worden.

Das Durchschnittsalter lag bei 72 Jahren (60-90 Jahren).15 Patienten gaben ein Trauma innerhalb der letzten 40 Tage an, 4 Patienten konnten sich nicht an ein Trauma erinnern. Die Lokalisation der Frakturen betraf bis auf jeweils eine Fraktur des 6. und des 8. Brustwirbelkörpers ausschließlich den thorakolumbalen Übergang. Sämtliche Frakturen waren dem Typ A nach Magerl et al. [17] zuzuordnen.

Bei 14 Patienten wurde Kalziumphosphat (durchschnittlich $8,0 \mathrm{ml}$ ), bei den übrigen 8 PMMA ( $7,5 \mathrm{ml})$ injiziert.

Unabhängig vom jeweils verwendeten Zement kam es zu folgenden intraoperativen Komplikationen: In einem Fall platzte ein in situ befindlicher Ballon während der Insufflation mit Kontrastmittel, in einem weiteren Fall wurde bei sechsgliedriger LWS zunächst der falsche Wirbelkörper augmentiert.

Bei der ersten Nachuntersuchung 3 Tage postoperativ äußerten 16 von $19 \mathrm{~Pa}$ -

Trauma Berufskrankh 2004 - 6 [Suppl 2] : S273-S278

DOI 10.1007/s10039-003-0793-1

(c) Springer-Verlag 2003

\section{T.R. Blattert · A. Weckbach}

Kalziumphosphat vs. Polymethylmethacrylat. Erste Ergebnisse einer prospektiven, randomisierten, klinischen Vergleichsstudie zur perkutanen Ballonkyphoplastie

\section{Zusammenfassung}

Derzeitiger Zementstandard für die Kypho-/Vertebroplastie ist Polymethylmethacrylat (PMMA), das jedoch durch fehlende ossäre Integrationsfähigkeit und eingeschränkte Biokompatibilität gekennzeichnet ist. Die vorliegende prospektive, randomisierte Studie überprüft die Eignung von Kalziumphosphat zur Augmentation thorakolumbaler Wirbelkörperfrakturen im Rahmen der perkutanen Ballonkyphoplastie und vergleicht sie mit einer PMMA-Kontrollgruppe. 22 Wirbelkörperfrakturen bei 19 Patienten wurden eingeschlossen. 14-mal wurde Kalziumphosphat, 8-mal PMMA injiziert. Postoperativ äußerten 16 Patienten eine Schmerzlinderung. Die durchschnittliche Aufrichtung des Grund-/Deckplattenwinkels betrug $5,4^{\circ}$. Ein statistisch signifikanter Unterschied zwischen beiden Gruppen wurde

nicht nachgewiesen. Zementspezifische Komplikationen waren für PMMA Gefäßembolisation $(n=2)$ sowie für Kalziumphosphat partielles „Auswaschen" des Zements ( $n=1)$ und radiologischer Korrekturverlust $(n=3)$ durch Zementversagen. Der Einsatz von Kalziumphosphat für die Kyphoplastie kann derzeit nicht generell empfohlen werden. Aufgrund der im Vergleich zu PMMA reduzierten Widerstandskraft gegen Biege-, Zugund Scherkräfte besteht ein u. U erhöhtes Risiko für Zementversagen und damit für einen postoperativen Korrekturverlust.

\section{Schlüsselwörter}

Kalziumphosphat · Kyphoplastie ·

Polymethylmethacrylat · Vertebroplastie . Wirbelkörper

\section{Calcium phosphate versus polymethylmethacrylate. Preliminary results of a prospective, randomized, clinical trial of percutaneous balloon kyphoplasty}

\section{Abstract}

In kyphoplasty and vertebroplasty, polymethylmethacrylate (PMMA) currently represents the standard augmentation material. It is characterized, however, by a lack of osteointegration and limited biocompatibility. This prospective, randomized trial investigated the feasibility of calcium phosphate (CaP) for augmentation of vertebral body fractures by percutaneous balloon kyphoplasty in comparison to PMMA. A total of 22 fractures in 19 patients were included whereby CaP was used in 14 and PMMA in 8 cases. Of these patients, 16 experienced pain relief. Endplate angles were restored by $5.4^{\circ}$ on average. No statistically significant difference was found between the groups. Some complications encountered were cement-specific such as vascular embolism ( $n=2)$ using PMMA, subtotal "cement-washout" $(n=1)$ and radiographic loss of correction ( $n=3$ ) due to cement failure using CaP. Currently in kyphoplasty, a routine use of $\mathrm{CaP}$ cannot be recommended. Due to its minor resistance to bending, extension, and shear forces compared to PMMA, there seems to be a higher risk for cement failure and subsequent loss of correction.

\section{Keywords}

Calcium phosphate $\cdot$ Kyphoplasty

Polymethylmethacrylate · Vertebral body . Vertebroplasty 

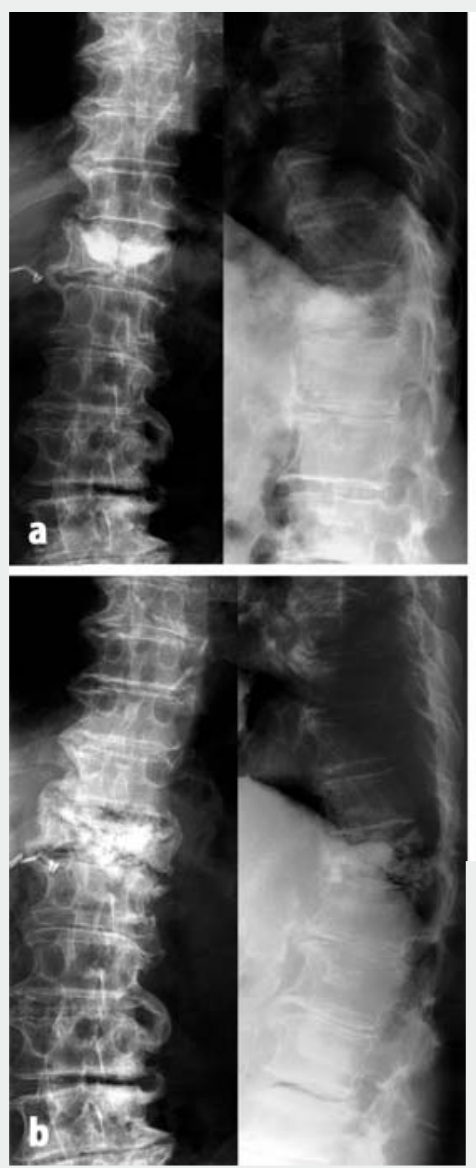

Abb. 3a-e $<$ Thorakolumbaler Übergang 3 Tage nach perkutaner, bipedikulärer Ballonkyphoplastie L1 mit Kalziumphosphat, Röntgen nativ a.-p. und seitlich im Stehen (a). Dieselbe Situation 6 Wochen postoperativ: deutlicher Korrekturverlust aufgrund ausgeprägter Zementfragmentierung (b). Axiale CTSchichten durch L1 (kranialer Anteil) zum Unfallzeitpunkt: sichtbare Zerstörung der Deckplatte mit Hinterkantenbeteiligung (A3-Verletzung) (c). Axiale CT-Schicht durch L1 3 Tage postoperativ: homogene Zementplombe in situ (d). Dieselbe Schnitthöhe 6 Wochen postoperativ: ausgeprägte Zementfrakturierung mit teilweiser Fragmentation und fortgeschrittener Resorption des Débris (e)

tienten eine deutliche subjektive Schmerzlinderung um mindestens 2 Notenstufen, wobei sich kein statistisch signifikanter Unterschied zwischen den beiden Randomgruppen nachweisen ließ. Die durchschnittliche Aufrichtung des bisegmentalen Grund-/Deckplattenwinkels betrug $5,4^{\circ}$. Die Wirbelkörpervorderkante konnte durchschnittlich um $7,5 \mathrm{~mm}$, die Hinterkante um 3,0 mm aufgerichtet werden, wobei bei 5 Patienten keine Aufrichtung gelang. Auch hier ließ sich kein Unterschied zwischen den beiden Randomgruppen erkennen.

\section{Zementbedingte Komplikationen}

\section{Zementextrusion}

Insgesamt 9 der 22 augmentierten Wirbelkörper zeigten eine stattgehabte Extrusion von Zement im postoperativ durchgeführten CT. Dabei kam es 5-mal zu einer Zementleckage außerhalb des Spinalkanals, wobei in allen 5 Fällen Kalziumphosphat verwendet wurde. Insgesamt 4-mal erfolgte ein Zementaustritt nach epidural, wobei es sich hier jedes Mal um PMMA handelte. In keinem Fall kam es jedoch zu neurologischen Ausfällen.

\section{Gefäßembolisation}

In insgesamt 2 Fällen kam es zu einer bereits intraoperativ erkennbaren Embolisation eines venös dränierenden Segmentalgefäßes (• Abb. 1), was jedoch keine klinischen Auswirkungen hatte. Beide Male kam PMMA zur Anwendung.

\section{Aushärtungsverhalten}

Bei anhaltender Blutung aus dem Wirbelkörper während der Zementapplikation kam es unter Verwendung von Kalziumphosphat in einem Fall zu einem partiellen „Auswaschen“ des Zements, noch bevor dieser aushärten konnte (• Abb. 2).

\section{Mechanische Eignung}

Zum Zeitpunkt der vorliegenden Zwischenerhebung hatten von den insgesamt 22 Wirbelkörpern 18 die 2. postoperative
Evaluation (6 Wochen postoperativ) abgeschlossen. 3 dieser 18 kurzfristig nachuntersuchten Wirbelkörper zeigten im seitlichen Nativröntgenbild nach 6 Wochen einen durch Zementversagen verursachten Verlust der intraoperativ erreichten Korrektur (• Abb. 3). Alle 3 Fälle hatten sowohl den Frakturtyp (A3) als auch das Augmentationsmaterial (Kalziumphosphat) gemeinsam.

\section{Diskussion}

Das traditionell verwendete Polymethylmethacrylat kann derzeit ohne Zweifel (noch) als das Standardaugmentationsmaterial für die Kypho- und Vertebroplastie angesehen werden $[2,10,11,27]$. Theoretisch stellen demgegenüber Biomaterialien auf Kalziumphosphatbasis, sofern sie in einer injizierbaren Applikationsform hergestellt werden können, aufgrund ihrer uneingeschränkten Biokompatibilität sowie ihres osteokonduktiven und biointegrativen Verhaltens eine ernst 
zu nehmende Alternative dar. Das Ziel kann dann nicht mehr Langzeitpräservation einer sich inert verhaltenden PMMAZementplombe sein, sondern Restrukturierung und anschließendes ossäres Remodelling des ehemals gebrochenen Wirbelkörpers - aus biologischer Sicht ein Gewinn. Zudem stellt die isotherme Aushärtung des letzteren Werkstoffs gegenüber der exothermen Polymerisation von PMMA auf dem Hintergrund verfahrensimmanenter und damit unvermeidlicher Zementextrusionen einen Vorteil dar.

Dabei ist allerdings noch unklar, ob Kalziumphosphat - neben den genannten theoretischen Vorteilen - auch die geforderten biomechanischen Fähigkeiten aufweist, um das diesbezügliche Leistungsvermögen von PMMA zumindest $\mathrm{zu}$ erreichen, wenn nicht zu übertreffen. Der biologische Vorteil allein rechtfertigt die Substitution von PMMA durch Kalziumphosphat jedenfalls nicht, wenn nicht gleichzeitig adäquate biomechanische Eigenschaften vorliegen.

Viele der eingangs postulierten theoretischen Vorbemerkungen scheinen sich im Verlauf der vorgelegten Studie zu bestätigen, wenngleich zum gegenwärtigen Zeitpunkt noch keine statistisch gesicherten Aussagen gemacht werden können. So zeigt sich, dass die Parameter Röntgendichte, Bearbeitungszeit und Aushärtungszeit tatsächlich bei beiden Materialgruppen bereits ideal gelöst und somit unproblematisch sind.

Die Bedenken hinsichtlich reduzierter Biokompatibilität von PMMA durch nicht polymerisierte toxische Monomere entwickelten in der bisherigen Serie keine klinische Relevanz. Etwa zu erwartende systemische Nebenwirkungen wie akute kardiopulmonale Reaktionen traten bislang bei der Applikation von PMMA nicht auf, was auch auf die geringe Rate von 1/22 Gefäßembolisationen durch den Zement zurückzuführen sein dürfte. Insofern scheint die Toxizität verbleibender PMMA-Monomere ähnlich der Embolisation eher ein Problem der Vertebroplastie mit ihren wesentlich höheren Arbeitsdrücken [8] als eines der Kyphoplastie zu sein.

Für die Beobachtung, dass sämtliche intraoperative PMMA-Extrusionen bisher ausschließlich nach epidural erfolg- ten, gleichzeitig aber alle Kalziumphosphataustritte außerhalb des Spinalkanals stattfanden, haben wir keine Erklärung. Wir scheuen uns auch, dies nach den bisherigen Ergebnissen als Trend zu betrachten und werden die definitive Auswertung nach Abschluss aller Nachuntersuchungen abwarten. Sollte sich ein Trend jedoch bestätigen, bekäme die Tatsache der exothermen Aushärtung von PMMA allerdings zusätzliche Brisanz.

Ob sich das Problem der vermehrten „Auswaschbarkeit“ von Kalziumphosphat gegenüber PMMA als klinisch relevant erweist, dürfte sich nach Abschluss der vorliegenden Untersuchung zeigen. Tatsache ist jedoch, dass es in einem Fall zu einem fast vollständigen Auswaschen von Kalziumphosphat durch anhaltende Blutung aus der Wirbelkörperspongiosa kam, mit der Konsequenz des gänzlich fehlenden Operationserfolgs. Unseres Wissens ist eine ähnliche Komplikation mit PMMA bislang noch nicht beschrieben.

Für die vorliegende Indikation bieten Kalziumphosphatzemente genügend axiale Kompressionsfestigkeit, da diese in etwa der physiologischen Festigkeit gesunder Wirbelkörperspongiosa entspricht [19]. Das Versagen der intrakorporellen Kalziumphosphatmasse in 3/14 Fällen der vorliegenden Untersuchung lässt sich nur dadurch erklären, dass hier neben axialer Kompression auch Kraftvektoren im Sinn der seitlichen Biegung, des Zugs und der Scherung nach intrakorporell fortgeleitet wurden, was nur bei Zerstörung der strukturellen Integrität des kortikalen Wirbelkörperrahmens denkbar ist. Dementsprechend gehören alle 3 betroffenen Wirbelkörperbrüche dem Typ A3 (Berstungsbruch) nach Magerl et al. [17] an und weisen damit per definitionem eine Endplattendestruktion mit begleitender Zerstörung der Wirbelkörperhinterkante auf. Ganz offensichtlich ist das Kalziumphosphat in dieser Situation den einwirkenden biomechanischen Kräften nicht gewachsen, sodass Biege-, Zug- und Scherkräfte zu einer frühzeitigen Frakturierung des Zements mit nachfolgender Fragmentierung und schließlich Korrekturverlust führen. Damit bestätigt sich, was für die interkorporelle Anwendung von Kalziumphosphat aus tierexperimentellen Untersuchungen bereits bekannt war [5].
Demgegenüber ist die typischste (und häufigste) osteoporotische Bruchform, die Wirbelkörperimpaktion (Typ A1.3), durch bloße, häufig grund- und deckplattenparallele Sinterung ohne weitergehende Destruktion der Endplatten gekennzeichnet. Erwartungsgemäß hielten die Kalziumphosphatplomben dieser Wirbelkörper dem - hier ausschließlich in axialer Belastungsrichtung einwirkenden - Kraftfluss stand. Da PMMA eine um den Faktor 10 höhere Widerstandskraft gegen Biege-, Zug- und Scherkräfte besitzt als der verwendete Kalziumphosphatzement, war ein Zementversagen durch biomechanische Beanspruchung hier nicht zu erwarten - auch nicht bei der A3-Verletzung, was sich ja in den Ergebnissen widerspiegelt.

\section{Ausblick: Zementoptimierung}

Osteokonduktive Biomaterialien auf Kalziumphosphatbasis sind aufgrund ihrer strukturellen Nähe zur originären Knochensubstanz hervorragend geeignet, das für die Kypho- und Vertebroplastie zurzeit meist verwendete Polymethylmethacrylat über kurz oder lang zu verdrängen. Viele der geforderten physiko-chemischen und biomechanischen Probleme sind bereits gelöst und entsprechende Anforderungen erfüllt. Es verbleiben allerdings die beiden für Kalziumphosphat spezifischen Probleme der erhöhten „Auswaschbarkeit“ und der geringen Widerstandsfähigkeit gegen Biegung, Zug und Scherung.

Die Möglichkeit einer Reduktion des „Auswasch“-Effekts wird derzeit experimentell durch verschiedene Additiva (Hydroxypropylmethylzellulose, Karboxylmethylzellulose, Chitosan) erprobt [7].

Beim Versuch der Verbesserung biomechanischer Eigenschaften von Kalziumphosphatzementen werden zurzeit 2 verschiedene Wege beschritten:

1. Die Möglichkeit einer strukturellen Primärstabilisierung durch Faserverstärkung (Aramid, Karbon, Bioglas) wird überprüft [12].

2. Durch Anreicherung der Kalziumphosphatgrundsubstanz mit osteoinduktiven Substanzen (BMP-2, BMP-7) wird eine frühzeitige In-vivo- 
Kallusumscheidung der Zementmasse - und damit deren Sekundärstabilisierung - angestrebt [4].

\section{Hinweis für die Praxis}

Der Einsatz von Zementen auf Kalziumphosphatbasis zur Wirbelkörperaugmentation bei der perkutanen Ballonkyphoplastie kann derzeit nicht generell empfohlen werden. Vielmehr besteht aufgrund der im Vergleich zu Polymethylmethacrylat um den Faktor 10 reduzierten Widerstandskraft gegen Biege-, Zug- und Scherkräfte ein erhöhtes Risiko für das Versagen der eingebrachten Zementplombe und damit für einen postoperativen Korrekturverlust. Dies scheint nach dem gegenwärtigen Stand insbesondere für Frakturen des Typs A3 zuzutreffen.

\section{Korrespondierender Autor Priv.-Doz.Dr. T. R. Blattert}

Unfall- \& Wiederherstellungschirurgie, Chirurgische Universitätsklinik Würzburg, Josef-Schneider-Straße 2, 97080 Würzburg, E-Mail: blattert@chirurgie.uni-wuerzburg.de

\section{Literatur}

1. Aebli N, Krebs J, Davis G et al. (2002) Fat embolism and acute hypotension during vertebroplasty. An experimental study in sheep. Spine 27: 460-466

2. Berlemann U, Heini PF (2002) Perkutane Zementierungstechniken zur Behandlung osteoporotischer Wirbelkörpersinterungen. Unfallchirurg 105: 2-8

3. Beruto DT, Mezzasalma SA, Capurro M et al. (2000) Use of $\alpha$-tricalcium phosphate (TCP) as powders and as an aqueous dispersion to modify processing, microstructure, and mechanical properties of polymethylmethacrylate (PMMA) bone cements and to produce bone-substitute compounds.J Biomed Mater Res 49: 498-505

4. Blattert TR, Delling G, Dalal PS et al. (2002) Successful transpedicular lumbar interbody fusion by means of a composite of osteogenic protein-1 (rhBMP-7) and hydroxyapatite carrier. A comparison with autograft and hydroxyapatite in the sheep spine. Spine 27: 26972705

5. Blattert TR, Delling G, Weckbach A (2003) Evaluation of an injectable calcium phosphate cement as an autograft substitute for transpedicular lumbar interbody fusion: controlled, prospective study in the sheep model. Eur Spine J 12: 216-23

6. Breed AL (1974) Experimental production of vascular hypotension, and bone marrow and fat embolism with methylmethacrylate cement. Traumatic hypertension of bone. Clin Orthop 1974: 227-244

7. Chow LC, Takagi S (2001) A natural bone cement A laboratory novelty led to the development of revolutionary new biomaterials. J Res Natl Inst Stand Technol 106: 1029-1033

8. Cortet B, Cotten A, Boutry N et al. (1999) Percutaneous vertebroplasty in the treatment of osteoporotic vertebral compression fractures: an open prospective study. J Rheumatol 26: 2222-2228
9. Deramond H, Wright NT, Belkoff SM (1999) Temperature elevation caused by bone cement polymerization during vertebroplasty. Bone [Suppl] 25: 17-21

10. Fourney DR, Schomer DF, Nader R et al. (2003) Percutaneous vertebroplasty and kyphoplasty for painful vertebral body fractures in cancer patients.J Neurosurg 98 : 21-30

11. Garfin SR, Yuan HA, Reiley MA (2001) New technologies in spine. Kyphoplasty and vertebroplasty for the treatment of painful osteoporotic compression fractures. Spine 26: 1511-1515

12. Gisep A (2002) Research on ceramic bone substitutes: current status. Injury 33: S-B88-92

13. Konno S, Olmarker K, Byrod G et al. (1994) The European Spine Society AcroMed Prize 1994: acute thermal nerve root injury. Eur Spine J 3: 299-302

14. Lee B-J, Lee S-R, Yoo T-Y (2002) Paraplegia as a complication of percutaneous vertebroplasty with polymethylmethacrylate. A case report. Spine 27: E419-E422

15. Lieberman IH, Dudeney S, Reinhardt M-K, Bell G (2001) Initial outcome and efficacy of "kyphoplasty" in the treatment of painful osteoporotic vertebral compression fractures. Spine 26: 1631-1638

16. Lim T-H, Brebach GT, Renner SM, Kim W-J et al. (2002) Biomechanical evaluation of an injectable calcium phosphate cement for vertebroplasty. Spine 27: 12971302

17. Magerl F, Aebi M, Gertzbein D et al. (1994) A comprehensive classification of thoracic and lumbar injuries. Eur Spine J 3: 184-201

18. Nakano M, Hirano N, Matsuura K et al. (2002) Percutaneous transpedicular vertebroplasty with calcium phosphate cement in the treatment of osteoporotic vertebral compression and burst fractures. J Neurosurg 97: 287-293

19. Norian Corporation. Norian SRSTM Skeletal Repair System, Cupertino, CA, USA (Produktbroschüre)

20. Padovani B, Kasriel O, Brunner P, Peretti-Viton P (2001) Pulmonary embolism caused by acrylic cement: a rare complication of percutaneous vertebroplasty. Am J Neuroradiol 20: 375-377

21. Peebles DJ, Ellis RH, Stride SD, Simpson BR (1972) Cardiovascular effects of polymethylmethacrylate cement. BMJ 1: 349-351

22. Phillips FM, Wetzel FT, Lieberman I, Campbell-Hupp M (2002) An in vivo comparison of the potential for extravertebral cement leak after vertebroplasty and kyphoplasty. Spine 27: 2173-2179

23. Phillips H, Cole PV, Lettin AW (1971) Cardiovascular effects of implanted acrylic bone cement. Bone Miner J 3: 460-461

24. Ratliff J, Nguyen T, Heiss J (2001) Root and spinal cord compression from methylmethacrylate vertebroplasty. Spine 26: E300-E302

25. Verlaan JJ, Helden WH van, Öner FC et al. (2002) Balloon vertebroplasty with calcium phosphate cement augmentation for direct restoration of traumatic thoracolumbar vertebral fractures. Spine 27: 543-548

26. Wilkes RA, Mackinnon JG, Thomas WG (1994) Neurological deterioration after cement injection into a vertebral body. J Bone Joint Surg Br 76: 155

27. Wong W, Reiley MA, Garfin S (2000) Vertebroplasty/Kyphoplasty.J Women Imag 2: 117-124 